\title{
Arabic gum acacia improves diabetic peripheral neuropathy in rats: a biochemical and histopathological evidence
}

\author{
Hailah M. Almohaimeed ${ }^{1 *}$, Hanan A. Amin ${ }^{2,3}$, Gamal S. Abd El-Aziz ${ }^{2}$, Hamed A. Saleh ${ }^{2}$
}

${ }^{1}$ Princess Nora Bint

Abdulrahman University (PNU), Riyadh. King Abdulaziz university (KAU), Jeddah, Saudi Arabia

${ }^{2}$ Department of Anatomy, Faculty of Medicine, King Abdulaziz University, Jeddah, Saudi Arabia

${ }^{3}$ Department of Histology, Faculty of Medicine, Cairo University, Egypt

Received: 11 April 2018

Accepted: 23 April 2018

\section{*Correspondence to:}

Dr. Hailah M. Almohaimeed, Email: Hailahmohammed.md@ gmail.com

Copyright: (C) the author(s), publisher and licensee Medip Academy. This is an openaccess article distributed under the terms of the Creative Commons Attribution NonCommercial License, which permits unrestricted noncommercial use, distribution, and reproduction in any medium, provided the original work is properly cited

\begin{abstract}
Background: Diabetic peripheral neuropathy (DPN) is a frequent complication of diabetes mellitus and unfortunately, its present therapeutic alternatives are exceptionally poor. Objectives of this study was to assess the antidiabetic, antioxidant and hypolipidemic action of Gum Arabic (GA) and its role in promoting the functional recovery from diabetic neuropathy developed in in an experimental model of diabetic neuropathy.

Methods: Sixty adult male Sprague-Dawley rats were utilized and randomly assigned into six groups $(n=10)$; control, Arabic gum-treated, untreated diabetic, diabetic received metformin, diabetic received metformin and B12 vitamin and diabetic received metformin, B12 vitamin and AG. Locomotor activity and hyperalgesia were assed at the end of the study. Fasting and two hours postprandial blood glucose, serum insulin levels, lipid Profile, oxidants/antioxidants parameters were assessed in the blood. Sciatic nerve was assessed histopathologically.

Results: The locomotor activity of the untreated diabetic rats was significantly $(\mathrm{p}<0.001)$ reduced compared to the control group while it was significantly increased in all treated groups. The lipid profile and Malondialdehyde were significantly improved in all treated groups. Levels of CAT, GSH, SOD, GPx were significantly decreased in untreated diabetic group compared to the control while they were significantly increased in all treated groups compared to the untreated diabetic group. Sciatic nerve fibers of untreated diabetic rats showed degenerated axons with dilated myelin sheaths and degenerated Schwann cells. The nerve had significantly fewer fiber compared to the control. These changes were alleviated in all the treated groups specifically that received metformin, vitamin B12 and GA.

Conclusions: It could be concluded that Arabic gum had hypoglycemic, antioxidant and hypolipidemic activity and had a protective effect on diabetic neuropathy. Based on this it is recommended that human clinical trials are necessary to prove this therapeutic effect.
\end{abstract}

Keywords: Arabic gum, Antioxidants, Diabetes melletus, Histology, Neuropathy, Sciatic nerve

\section{INTRODUCTION}

Diabetes mellitus (DM) is thought to be a noteworthy risk to public health. Data from the World Health Organization (WHO) showed that almost half of all deaths attributable to high blood glucose occur before the age of 70 years. WHO projects that diabetes will be the seventh leading cause of death in 2030. ${ }^{1}$ Diabetic peripheral neuropathy $(\mathrm{DPN})$ is a frequent complication of diabetes mellitus.
Studies conducted in the Middle East revealed high rates of DPN ranged from $35 \%$ to $65 \%$. The DPN is more frequently represented than other complications of Diabetes Mellitus. It was reported that it is responsible for $50 \%-75 \%$ of non-traumatic amputations. Painful DPN has high grade of functional disability which influences health-related quality of life. ${ }^{2}$ Hyperglycemia is basic for occurrence and advancement of DPN with the etiological hypotheses on metabolic or vascular causes. ${ }^{3}$ Regardless of numerous investigations on DPN, the present 
therapeutic alternatives are exceptionally poor. Neuropathy contributes the greatest morbidity and mortality and extremely disables because of parasthesia and neuropathic injury, the direct cause of amputation. ${ }^{4}$ DPN could cause atrophy, demyelination of nerve axons. ${ }^{5}$

Alternative medicine is commonly used in developing countries up to $80 \% .^{1}$ Acacia gum (AG) is known as a non-absorbable polysaccharide with very low glycaemic index. It is extracted from trees of Acacia senegal and Acacia seyal in Africa. ${ }^{6}$ No adverse effects were recorded after AG use even at very high doses. ${ }^{7}$ Intestinal bacteria fermentation transforms Arabic gum to fatty acids that is considered a prebiotic. It prevents glucose absorption, and so reduce plasma glucose by elevating the insulin secretion. ${ }^{8}$ Gum Acacia, is traditionally used in the treatment diabetes. ${ }^{9}$

Hyperglycemia induced oxidative stress can be the cause of diabetic neuropathy, that could be treated by antioxidants. Arabic gum is considered as anti-diabetic, anti-lipidemic, antioxidant and anti-inflammatory. Therefore, this study aimed to assess the anti-diabetic, anti-oxidant and hypolipidemic action of GA and its role in promoting the functional recovery from diabetic neuropathy developed in in an experimental model of diabetic neuropathy.

\section{METHODS}

\section{Chemicals}

Arabic Gum was obtained from local supermarket as instant gum powder granules from Elnasr for food industries. Streptozotocin (STZ) and Metformin hydrochloride was obtained from Merck Company and vitamin B12 cyanocobalamin was obtained from Eisai Company. Induction of DM was done using intra-peritoneal injection of STZ once at the dose of $60 \mathrm{mg} / \mathrm{Kg} .{ }^{10}$ Metformin was administered at the dose $300 \mathrm{mg} / \mathrm{kg}$ according to Mennatallah et al. ${ }^{11}$ Vitamin B12 was administered at the dose $1.77 \mathrm{mg} / \mathrm{kg}$ according to Hosseinzadeh. ${ }^{12}$ Arabic Gum was administered at the dose of $4 \mathrm{gm} / \mathrm{kg}$. ${ }^{13}$

\section{Animals and experimental design}

Sixty adult male Sprague-Dawley rats were purchased from the animal unit at King Fahd Medical Research Center, King Abdulaziz University, Jeddah, KSA. Animal procedures were conducted in accordance with the Committee of Animal Investigations, Faculty of Medicine, King Abdulaziz University.

Rats were randomly assigned into six groups $(n=10)$; the control group (G I) which received the standard diet plus the vehicle, Arabic gum group (G II) which received the standard diet plus AG aqueous extract. The untreated diabetic group (G III) which received no treatment after induction of DM. The diabetic group treated with metformin (G IV), the diabetic group treated with metformin and B12 vitamin $(\mathrm{G} \mathrm{V})$ and the diabetic group treated with metformin, B12 vitamin and AG (G VI). Vehicle and all treatment were started three weeks after the induction of DM and continued for eight consecutive weeks.

\section{Behavioral tests}

After eight weeks of treatment, assessment of locomotor activity was done using the open field test. Rats set on the focal square and when rat entered a square with four legs, the quantity of squares that were crossed was recorded in a 5 minutes session.

Hot plat test was done to assess the hyperalgesia. The hyperalgesic reaction on the hot-plate composed of a blend of focal and fringe components. In this test, rats independently were set on a hotplate with the temperature acclimated to $55 \pm 1^{\circ} \mathrm{C}$. The inertness to the primary indication of paw licking or hop reaction to maintain a strategic distance from the warmth was be taken as a record of the torment edge; the cut-off time was $10 \mathrm{~s}$ keeping in mind the end goal to evade harm to the paw.

\section{Biochemical assessment}

After finishing the behavioral testes, blood samples were drawn from tail vein. They were store for estimation of fasting and two hours post prandial blood glucose levels, serum insulin level, lipid Profile (Total cholesterol, High density lipoproteins (HDL-C), very low density lipoproteins (vLDL-C), low density lipoproteins (LDL-C) and triglyceride levels), and oxidative stress parameter; Malondialdehyde (MDA) as well as the antioxidants parameters Superoxide dismutase (SOD), catalase (CAT), and Glutathione peroxidase (GPx) and reduced glutathione (GSH).

\section{Histopathological assessment}

At the end of the experiment, rats were sacrificed, the lower limbs were dissected out to isolate the sciatic nerve. The latter was fixed in $10 \%$ neutral buffered formalin and processed for obtaining paraffin blocks and sections to be stained with Hematoxylin and eosin ( $\mathrm{H}$ and $\mathrm{E})$.

\section{Statistical analysis}

Statistical studies were done using statistical package of social science (SPSS). Results were represented as mean \pm standard error (SE).

\section{RESULTS}

\section{Behavioral changes}

The locomotor activity of animals were assessed. It was noticed that the total distance traveled by the rats was significantly reduced $(\mathrm{p}<0.001)$ in untreated diabetic rats compared with the control rats. On the other hand, the total travel distance was significantly increased in G IV 
$(\mathrm{p}=0.001), \mathrm{GV}(\mathrm{p}<0.001)$ and $(\mathrm{GVI}, \mathrm{p}<0.001)$ compared to diabetic untreated group, whereas the velocity was significantly reduced $(\mathrm{p}=0.003)$ in the untreated diabetic rats compared to the control group. Although, the velocity was increased in all the treated groups, this increase was significant only in $\mathrm{GV}(\mathrm{p}=0.013)$ compared to the diabetic untreated group (Table 1).

Table 1: Multiple comparisons between the distance test, velocity test and hot plate test for the control group and other groups.

\begin{tabular}{|lllll|}
\hline Groups & Distance test $(\mathbf{c m})$ & Velocity test $(\mathrm{cm} / \mathrm{sec})$ & $\begin{array}{l}\text { Hot Plate Test (sec) } \\
\text { before treatment }\end{array}$ & $\begin{array}{l}\text { Hot Plate Test (sec) } \\
\text { after treatment }\end{array}$ \\
\hline Group I & $3369 \pm 146$ & $3.08 \pm 0.051$ & $20 \pm 0.62$ & $20 \pm 0.62$ \\
\hline Group II & $3395 \pm 74$ & $3.07 \pm 0.100$ & $20 \pm 0.42$ & $19 \pm 0.54$ \\
\hline Group III & $1052 \pm 52^{*}$ & $1.9 \pm .215^{* \#}$ & $31 \pm 1.0^{*}$ & $29 \pm 1.0^{* \#}$ \\
\hline Group IV & $2215 \pm 182^{* \#}$ & $2.4 \pm 50^{*}$ & $32 \pm 3.0^{*}$ & $25 \pm 2.5^{\#} *$ \\
\hline Group V & $2712 \pm .0 .213^{\# *}$ & $2.8 \pm 0.454$ & $29 \pm 2.9^{*}$ & $22 \pm 1.4$ \\
\hline Group VI & $2327 \pm 0.196^{\# *}$ & $2.03 \pm 0.159^{*}$ & $28 \pm 0.64^{*}$ & $20 \pm 1.5$ \\
\hline
\end{tabular}

Values are represented as mean \pm SE. Significance with the control group. * Significance with the control group, \# Significance with diabetic group (Group III). GI (control), GII (Arabic gum group), GIII (untreated diabetic control group), GIV (diabetic + metformin), $\mathrm{GV}$ (diabetic + metformin + B12 vitamin) and (diabetic + metformin + B12 vitamin +Arabic gum acacia)

Table 2: Multiple comparisons between the fasting and after 2 hours blood glucose (BGL) levels (mg/dl) and serum insulin (uIU/ml) for the control group and other groups.

\begin{tabular}{|llll|}
\hline Groups & Fasting BGL $(\mathrm{mg} / \mathrm{dl})$ & BGL after 2 hours $(\mathrm{mg} / \mathrm{dl})$ & serum insulin $(\mathrm{uIU} / \mathrm{ml})$ \\
\hline Group I & $74 \pm 2.7$ & $112 \pm 4.7^{\#}$ & $76.6 \pm 1.4^{*}$ \\
\hline Group & $72 \pm 1^{\#}$ & $94 \pm 1.3^{* \#}$ & $92.2 \pm 2.0^{*}$ \\
\hline Group III & $169 \pm 33.5^{*}$ & $147 \pm 2.5^{*}$ & $38.4 \pm 2.1^{*}$ \\
\hline Group IV & $123 \pm 3^{*}$ & $133 \pm 50^{*}$ & $69.3 \pm 1.1{ }^{*}$ \\
\hline Group V & $116 \pm 4.4^{*}$ & $139 \pm 3.5^{*}$ & $70.8 \pm 0.80^{* \#}$ \\
\hline Group VI & $101 \pm 6.1 *$ & $122 \pm 3.9^{* \#}$ & $71.8 \pm 1.2^{\#}$ \\
\hline
\end{tabular}

Values are represented as mean \pm SE. Significance with the control group. *Significance with the control group, \# Significance with diabetic group (Group III). GI (control), GII (Arabic gum group), GIII (untreated diabetic control group), GIV (diabetic + metformin), $\mathrm{GV}$ (diabetic + metformin + B12 vitamin) and (diabetic + metformin + B12 vitamin +Arabic gum acacia).

Table 3: Multiple comparisons between the serum levels (mg/dl) of cholesterol; HDL-C (mg/dl); vLDLC; LDL-C; and Triglyceride for the control group and other groups.

\begin{tabular}{|llllll|}
\hline Groups & Total cholesterol & HDL-C & vLDL-C & LDL-C & Triglyceride \\
\hline Group I & $58.2 \pm 5.9 *$ & $86.9 \pm 2.0^{*}$ & $8.3 \pm 0.15$ & $12.0 \pm 0.05 *$ & $52.2 \pm 2.2$ \\
\hline Group II & $49.2 \pm 1.1 *$ & $119.4 \pm 1.2^{*}$ & $6.9 \pm 20^{*}$ & $9.2 \pm 0.75^{*}$ & $37.1 \pm 1.9 *$ \\
\hline Group III & $126.7 \pm 5.6 *$ & $39.5 \pm 0.54^{*}$ & $22.2 \pm 1.3^{*}$ & $24.9 \pm 0.65 *$ & $114.4 \pm 4.1 *$ \\
\hline Group IV & $71.6 \pm 1.4 * \#$ & $76.4 \pm 0.80^{* \#}$ & $8.9 \pm 0.05^{*} *$ & $13.4 \pm 0.4^{*}$ & $63.3 \pm 2.8^{*} \#$ \\
\hline Group V & $66.3 \pm 0.75^{* \#}$ & $77.0 \pm 1.0^{* \#}$ & $9.2 \pm 0.24^{*} *$ & $13.6 \pm 0.55^{\#}$ & $57.2 \pm 1.7 * \#$ \\
\hline Group VI & $63.2 \pm 0.90^{* \#}$ & $79.8 \pm 0.35^{* \#}$ & $8.9 \pm 0.10^{*} \#$ & $12.5 \pm 0.45^{\#}$ & $55.4 \pm 0.50^{* \#}$ \\
\hline
\end{tabular}

Values are represented as mean \pm SE. Significance with the control group. *Significance with the control group, \# Significance with diabetic group (Group III). GI (control), GII (Arabic gum group), GIII (untreated diabetic control group), GIV (diabetic + metformin), $\mathrm{GV}$ (diabetic + metformin + B12 vitamin) and (diabetic + metformin + B12 vitamin +Arabic gum acacia).

Upon assessing the thermal hyperalgesia using the hot plate test before starting the treatment, it was noticed that there was insignificant difference between the mean latency period between the untreated diabetic group and all treated groups. After treatment, thermal hyperalgesia was reassessed and there was significant decrease $(\mathrm{p}=0.002$ and $\mathrm{p}<0.001)$ in the latency period in the treated groups $(\mathrm{GV})$ and (GVI) respectively in comparison to the untreated diabetic group (Table 1).

\section{Biochemical results}

Fasting blood glucose was significantly decreased in the three treated groups when compared to the untreated diabetic one. There was no significant difference between 
any of the treated groups. When it came to the blood glucose level estimated after 2 hours, it was found that it significantly decreased in G II compared to the control group. Serum insulin level, in this study, was significantly elevated in the control group received GA compared with the control rats (Table 2).

When it came to the effect of GA on the lipid profile of the control rats, this study showed that there was significant decrease in triglycerides, VLDL-C, LDL-C levels in AGtreated group while HDL-C level significantly increase compared to the control group. Regarding the effect of GA on the lipid profile of diabetic rats, it was observed that there was significant increase in serum total cholesterol, triglycerides, VLDL-C, levels in the untreated diabetic group compared to the control group. On the other hand, the serum total cholesterol, triglycerides, VLDL-C and LDL-C levels were significantly decreased in all treated groups compared to untreated diabetic one with the maximum decrease in the group treated with the combination of metformin and vitamin B12 and Arabic gum (Table 3).

Serum level of MDA was assessed as a marker of oxidative stress process. It was noticed that that MDA significantly increased in untreated diabetic group compared to the control group while its level was significantly decreased in all treated groups compared to untreated diabetic group. The levels of CAT, GSH, SOD, GPx were significantly decreased in untreated diabeteic group compared to the control while they were significantly increased in all treated groups compared to the untreated diabetic group with the maximum increase in group treated with the combination of metformin and vitamin B12 and Arabic gum (Table 4).

Table 4: Multiple comparisons between the serum levels of Superoxide dismutase (SOD), catalase (CAT), Malondialdehyde (MDA) and Glutathione peroxidase (GPx) and reduced f glutathione (GSH).

\begin{tabular}{|c|c|c|c|c|c|}
\hline Group & CAT(U/L) & $\operatorname{MDA}(\mathrm{nmol} / \mathrm{ml})$ & SOD $(\mathrm{U} / \mathrm{ml}$ & GPx $(\mathrm{mU} / \mathrm{ml})$ & GSH(mg/dl) \\
\hline Groups & $338.2 \pm 16.9 *$ & $2.4 \pm 0.40$ & $87.6 \pm 5.2 *$ & $140 \pm 10$ & $13.2 \pm 0.70$ \\
\hline Group I & $320 . \pm 5.4$ & $2.1 \pm 0.7$ & $93.5 \pm 6.7$ & $126 \pm 22$ & $22.7 \pm 0.25 *$ \\
\hline Group & $77.6 \pm 0.50 *$ & $1.9 \pm 4.7$ & $15.9 \pm 0.86^{*}$ & $56 \pm 7.9 *$ & $8.0 \pm 0.49 *$ \\
\hline Group III & $225 \pm 8.3^{* \#}$ & $2.6 \pm 0.39^{\#}$ & $47.6 \pm 2.4$ *\# & $84 \pm 6.4^{*}$ & $15.1 \pm 1.7^{\#}$ \\
\hline Group IV & $218 \pm 4.2 * \#$ & $5.2 \pm 0.34^{\#}$ & $43.8 \pm 2.9$ *\# & $205 \pm 23 \#$ & $11.1 \pm 0.61$ \\
\hline Group V & $302 \pm 7.03^{* \#}$ & $2.8 \pm 0.45^{\#}$ & $59.3 \pm 3.9$ *\# & $104 \pm 4.0$ & $16.5 \pm 1.6^{\#}$ \\
\hline
\end{tabular}

Values are represented as mean \pm SE. Significance with the control group. *Significance with the control group, \# Significance with diabetic group (Group III). GI (control), GII (Arabic gum group), GIII (untreated diabetic control group), GIV (diabetic + metformin), $\mathrm{GV}$ (diabetic + metformin + B12 vitamin) and (diabetic + metformin + B12 vitamin +Arabic gum acacia).

\section{Histopathological results}

The light microscopic examination of $\mathrm{H}$ and E-stained sections of sciatic nerve of the control group. The sciatic showed normal structure and regular arrangement of its nerve fibers. The perineurium (connective tissue surrounded the nerve), nerve axons and Schwann cells appeared intact. The sciatic nerve of GII which received Arabic Gum had normal structure and arrangement that were nearly similar to the control group (Figure 1). On the other hand, the sciatic nerve of the untreated diabetic group showed less number of dispersed nerve fibers with smaller diameter compared to the control group. Some axons appeared degenerated with darkly stained cytoplasm. In other sections some axons appeared vacuolated and swollen with dilated myelin sheaths. Some Schwann cells possessed dark nuclei indicated its degeneration (Figure $1)$.

Sciatic nerve of GIV and GV showed increased number of nerve fibers compared to the untreated group. Most of the nerve fibers were regularly arranged. Few axons appeared degenerated with darkly stained cytoplasm (arrow) while most of the axons appear intact. Schwann cells appeared not affected. Sciatic nerve of GVI (diabetic rats treated with metformin plus Vitamin B12 and AG) showed that most of the nerve axons appear intact and regularly arranged apart from very few degenerated fibers. Schwann cells appeared not affected (Figure 1).

When it came to the morphometric assessment of the histopathological changes observed in the studied groups, it was noticed that there was significant decrease $(\mathrm{p}<0.001)$ in the diameter of the nerve fibers of sciatic nerve in the untreated diabetic group compared with the control rats. There was significant increase $(p<0.001)$ in nerve fiber diameter of all treated groups compared with untreated diabetic one (Figure 2).

\section{DISCUSSION}

Fasting blood glucose showed significant decrease in the three treated groups when compared with the untreated diabetic group. There was no significant difference between any of the treated groups. When it came to the blood glucose level estimated after 2 hours, it was found that it significantly decreased in G II compared to the 
control group. These findings were in concordance with those of Wadood et al. who recorded a significant reduction in blood glucose level after oral intake of aqueous extract of Arabic gum in normal rats. ${ }^{13}$

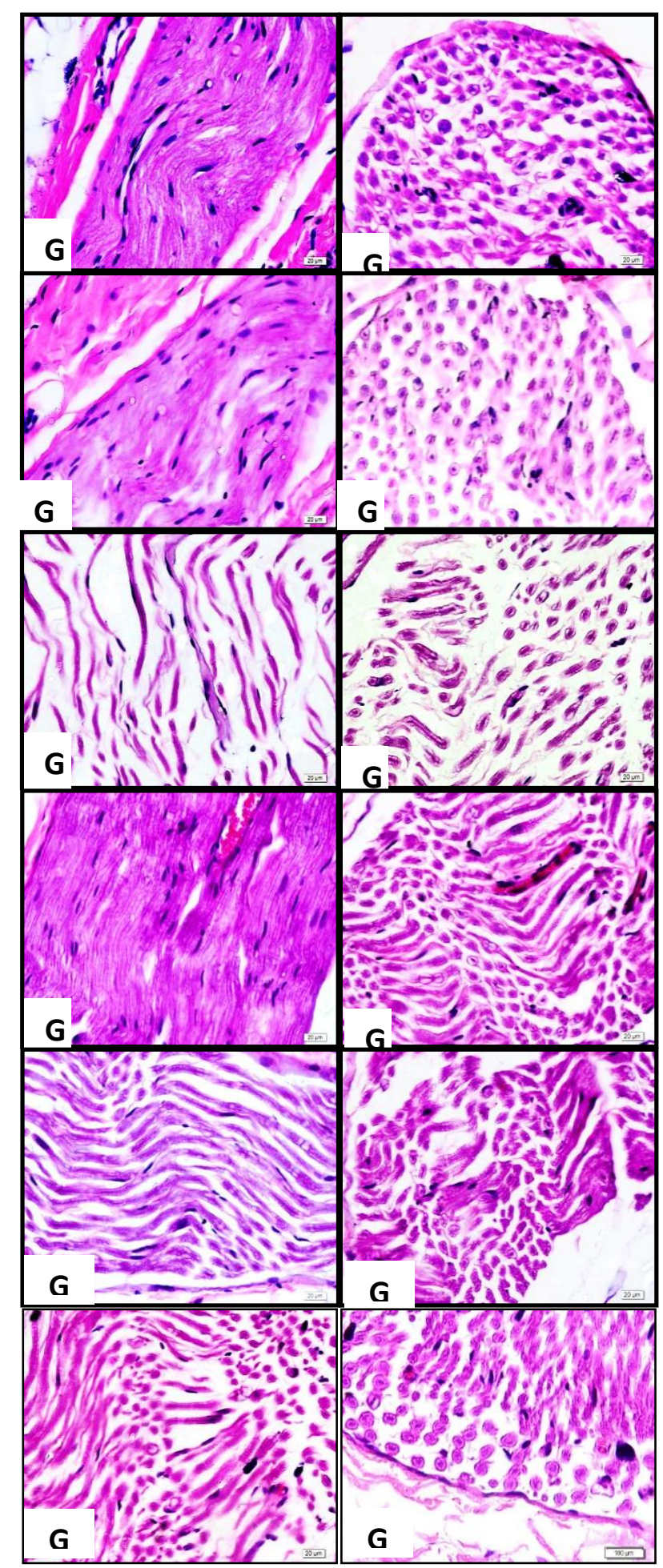

Figure (1): Shows the differences in siatic nerve histology between GI (control), GII (Arabic gum group), GIII (untreated diabetic control group), GIV (diabetic + metformin), GV (diabetic + metformin +

B12 vitamin) and (diabetic + metformin + B12 vitamin +Arabic gum acacia).

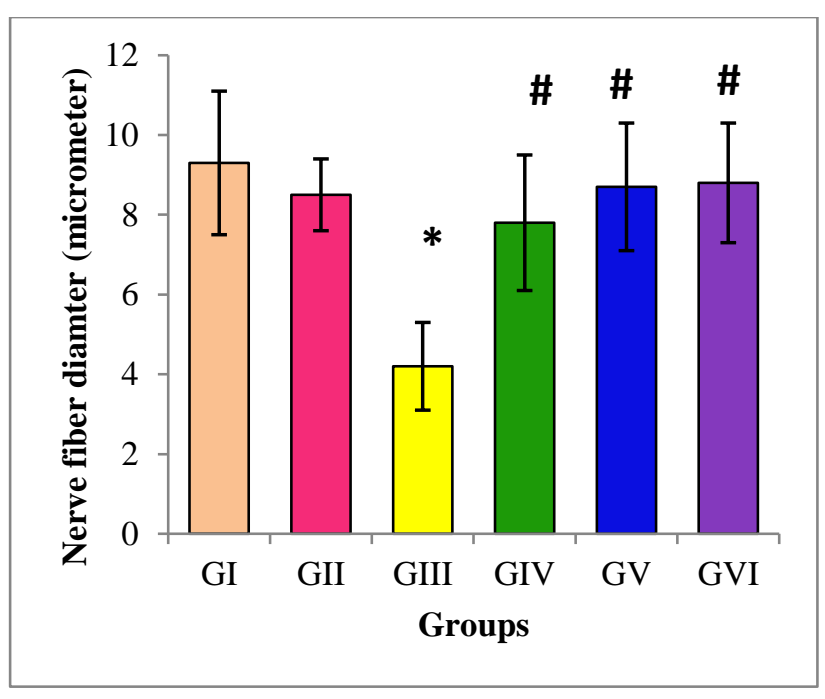

Figure (2): Diameter of the nerve fibers of sciatic nerve in all studied groups. * indicates significant change compared to the control group, while \#

indicated significant change compared to the untreated diabetic group. GI (control), GII (Arabic gum group), GIII (untreated diabetic control group),

GIV (diabetic + metformin), GV (diabetic + metformin + B12 vitamin) and (diabetic + metformin + B12 vitamin +Arabic gum acacia).

In another study, Arabic gum induced a significant hypoglycemic effect in non-diabetic rabbits while it could not significantly reduce blood sugar in alloxan-induced diabetic rabbits Hou et al. ${ }^{14}$ Serum insulin level, in this study, was significantly elevated in the control group received GA compared with the control one. In a previous study, Acacia Arabica-induced hypoglycemic effect in normal control rats was attributed to stimulated insulin secertion. ${ }^{15}$

When it came to the effect of GA on the lipid profile of the control rats, this study showed that there was significant decrease in triglycerides, VLDL-C, LDL-C levels in AGtreated group while HDL-C level significantly increase compared to the control group. This finding was supported by the results of Yasir et al, who reported both hypoglycemic and hypolipidemic effect of Acacia Arabica in control rats. ${ }^{15}$ On the other hand, these findings were contradicted with those of Hegazy et al, who reported that GA did not change lipid profile, in the normal control rats. ${ }^{16}$ This might to attributed to the duration of administration of the GA as Hegazy et al, gave GA for only three weeks while in this study, the extract of GA was administrated for 10 weeks. ${ }^{16}$ Reduced total serum cholesterol was reported in subjects given $25 \mathrm{~g} /$ day and $30 \mathrm{~g} /$ day of GA for 21 and 30 days respectively (Ross et al) The reduction was limited to LDL-C and VLDL-C only, with no effect on HDL-C and triglycerides.

In this study, administration of $\mathrm{AG}$ in combination with metformin and Vitamin B12, improved the lipid profile of diabetic rats. In an earlier study, aqueous extract of Arabic 
gum orally administrated at a dose of $400 \mathrm{mg} / \mathrm{kg}$ significantly decreased cholesterol, and triglycerides levels in diabetic rats. ${ }^{13}$ In some previous studies both hypolipidemic and hypoglycemic effects of Acacia Arabica extract were reported in different models of diabetic animals. ${ }^{15,17,18}$ Patil et al, reported that the chloroform extract of Acacia arabica bark reversed the cholesterol, triglyceride, and HDL and LDL values when compared to untreated diabetic rats. ${ }^{19}$ In a more recent study, the hypolipidemic effect of GA was confirmed by Hegazy et al, who concluded that Acacia Arabica extract administrated for 3weeks (100 and $200 \mathrm{mg} / \mathrm{kg})$ significantly reduce triglycerides, LDL-C, and total cholesterol and significantly increased HDL-C in diabetic rats. ${ }^{16}$

In this study, MDA significantly increased in untreated diabetic rats compared to the control one while its level was significantly decreased in all treated groups compared to untreated diabetic group. This result agreed with the result of Hegazy et al, who stated that Acacia Arabica extract significantly reduced MDA compared to the diabetic group. ${ }^{16}$ Increased MDA observed in diabetic patients was reported to be attributed to the chronic hyperglycemia that significantly changes oxidative stress markers. ${ }^{20}$ In addition, there was significant decrease in CAT, GSH, SOD, GPx levels in serum of untreated diabetic group compared to control one. In a previous study, significant depletion in GSH and GPx content were observed in diabetic patients in comparison with controls. This depletion was attributed to the chronic hyperglycemia. ${ }^{20}$ Hyperglycemia, which leads to cellular tissue damage, was previously reported to induce oxidative stress. ${ }^{21}$ In a previous study, Alloxan-induced diabetes caused significant decrease in SOD, CAT, and GPX activities as well as significant increase in MDA production. ${ }^{22}$ These changes reflect oxidative stress which may be mirror a decreased antioxidant defense potential. ${ }^{23}$

In this study sciatic nerve from untreated diabetic rats showed fewer number of dispersed nerve fibers with smaller diameter with some degenerated axons and dilated myelin sheaths. Some Schwann cells were degenerated. There was significant decrease in the diameter of the nerve fibers of sciatic nerve in untreated diabetic rats compared to the control group. These finding were in concordance with those of Reda et al, who observed degeneration in the nerve fibers with dilated myelin sheath and vacuolization with swollen axon in sciatic nerve from the diabetic rats. ${ }^{24}$ During his study on STZ-induced diabetes in rats, Afifi, observed marked axonal atrophy in sciatic nerve fibers along with axonal shrinkage and sometimes total axonal destruction. ${ }^{25}$ They observed a significant decrease in sciatic nerve fibers in the diabetic rats compared with the control. These findings were in agreement with the histopathological findings observed in this study. Unfortunately, there was no previous studies describing the effect of GA on the sciatic nerve histopathological changes occurred in diabetic neuropathy.

\section{CONCLUSION}

It could be concluded that Arabic gum had hypoglycemic, antioxidant and hypolipidemic activity and had a protective effect on diabetic neuropathy. Based on this it is recommended that human clinical trials are necessary to prove this therapeutic effect.

Funding: No funding sources

Conflict of interest: None declared

Ethical approval: The study was approved by the Institutional Ethics Committee

\section{REFERENCES}

1. World Health Organization. Prevalence of diabetes worldwide. 2017. Avalible at: http://www who int/diabetes/facts/world_figures/en/.

2. Janahi NM, Bakhiet M. Diabetic Peripheral Neuropathy: A Common Complication in Diabetic Patients Bahrain Medical Bulletin. 2015;37(1).

3. Diabetes Control and Complication Trial Research Group. The effect of intensive diabetes therapy on the development and progression of neuropathy. Ann Intern Med. 1995;122:561-8.

4. Benbow SJ, Wallymahmed ME, MacFarlane IA. Diabetic peripheral neuropathy and quality of life. QJM: monthly journal of the Association of Physicians. 1998 Nov 1;91(11):733-7.

5. Piao Y, Liang $X$. Chinese medicine in diabetic peripheral neuropathy: experimental research on nerve repair and regeneration. Evidence-Based Complementary and Alternative Medicine. 2012:1-3.

6. Maslin BR, Miller JT, Seigler DS. Overview of the generic status of Acacia (Leguminosae: Mimosoideae). Australian Systematic Botany. 2003;16(1):1-8.

7. Phillips GO, Ogasawara T, Ushida K. The regulatory and scientific approach to defining gum arabic (Acacia senegal and Acacia seyal) as a dietary fibre. Food hydrocolloids. 2008 Jan 1;22(1):24-35.

8. Philips AO, Philips GO. Biofunctional behavior and health benefits of a specific Gum Arabic. Food hydrocoll. 2011;25(2):165-9.

9. Meance SE. Acacia gum (Fibregum ${ }^{\mathrm{TM}}$ ), a very well tolerated specific natural prebiotic having a wide range of food applications-Part 1. AGRO FOOD INDUSTRY HI TECH. 2004 Jan 1;15(1):24-9.

10. Makino A, Kamata $K$. Effects of chronic administration of L-arginine on vasoactive responses induced by endothelin-1 and its plasma level in streptozotocin-induced diabetic rats. J Smooth Muscle Research. 2002;38(4, 5):101-15.

11. Ali MA, El-Abhar HS, Kamel MA, Attia AS. Antidiabetic effect of galantamine: novel effect for a known centrally acting drug. PloS one. 2015 Aug 11;10(8): 0134648.

12. Hosseinzadeh H, Moallem SA, Moshiri M, Sarnavazi MS, Etemad L. Anti-nociceptive and antiinflammatory effects of cyanocobalamin (vitamin 
B12) against acute and chronic pain and inflammation in mice. Arzneimittelforschung. 2012 Jul;62(7):324-9.

13. Wadood AB, Wadood NO, Shah SA. Effects of Acacia arabica and Caralluma edulis on blood glucose levels of normal and alloxan diabetic rabbits. JPMA. The Journal of the Pakistan Medical Association. 1989 Aug;39(8):208-12.

14. Hou CC, Lin SJ, Cheng JT, Hsu FL. Antidiabetic dimeric guianolides and a lignan glycoside from Lactuca indica. J. Nat. Prod. 2003;66(5):625-629.

15. Yasir M, Shrivastava R, Jain P, Das D, Yasir M, Shrivastava R, et al. Hypoglycemic and Antihyperglycemic Effects of Different Extracts and Combinations of Withania coagulans Dunal and Acacia arabica Lamk in Normal and Alloxan Induced Diabetic Rats. Pharmacognosy Communications. 2012; 2: 61-66.

16. Hegazy GA, Alnoury AM, Gad HG. The role of Acacia Arabica extract as an antidiabetic, antihyperlipidemic, and antioxidant in streptozotocininduced diabetic rats. Saudi medical journal. $2013 \mathrm{Jul}$ 1;34(7):727-33.

17. Liu X, Kim J, Li Y, Li J, Liu F, Chen X. Tannic acid stimulates glucose transport and inhibits adipocyte differentiation in 3T3-L1 cells. J Nutr. 2005;135:16571.

18. Ahmed M, Zaman F, Sharif T, Zabta ChM. Antidiabetic and hypolipidemic effects of aqueous methanolic extract of acacia nilotica pods in alloxaninduced diabetic rabbits. Scan J Lab Anim Sci. 2008;35:29-34.

19. Patil RN, Patil RY, Ahirwar B, Ahirwar D. Evaluation of antidiabetic and related actions of some Indian medicinal plants in diabetic rats. Asian Pacific J Tropical Med. 2011;4:20-33.
20. Aouacheri O, Saka S, Krim M, Messaadia A, Maidi I. The investigation of the oxidative stress-related parameters in type 2 diabetes mellitus. Can J Diabetes. 2015 Feb;39(1):44-9.

21. Gupta S, Kataria M, Gupta PK, Murganandan S, Yashroy RC. Protective role of extracts of neem seeds in diabetes caused by streptozotocin in rats. Int $\mathbf{J}$ Pharm. Ethnopharmacol. 2004 Feb;90(2-3):185-9.

22. Ahmed AA, Fedail JS, Musa HH, Kamboh AA, Sifaldin AZ, Musa TH. Gum Arabic extracts protect against hepatic oxidative stress in alloxan induced diabetes in rats. Pathophysiology. 2015 Dec;22(4):189-94.

23. Tiwari BK, Pandey KB, Abidi AB, Rizvi SI. Markers of oxidative stress during diabetes mellitus. Journal of biomarkers. 2013:1-8.

24. Reda HM, Zaitone SA, Moustafa YM. Effect of levetiracetam versus gabapentin on peripheral neuropathy and sciatic degeneration in streptozotocindiabetic mice: influence on spinal microglia and astrocytes. European J pharmacol. 2016 Jan 15;771:162-72.

25. Afifi NM. Neuroprotective effect of melatonin in a rat model of streptozotocin-induced diabetic neuropathy: Light and electron microscopic study. Egyp J Histology. 2013;36:321-35.

Cite this article as: Almohaimeed HM, Amin HA, Abd El-Aziz GS, Saleh HA. Arabic gum acacia improves diabetic peripheral neuropathy in rats: a biochemical and histopathological evidence. Int $\mathbf{J}$ Basic Clin Pharmacol 2018;7:1065-71. 